\title{
Differentiation of human amniotic fluid-derived mesenchymal stem cells into type II alveolar epithelial cells in vitro
}

\author{
YAQING LI ${ }^{1}$, WULIN XU ${ }^{1}$, JIANPING YAN ${ }^{1}$, YINGJIE XIA ${ }^{2}$, CHAO GU $^{1}$, YINGYU MA ${ }^{2}$ and HOUQUAN TAO ${ }^{2}$ \\ ${ }^{1}$ Department of Respiratory Medicine and ${ }^{2}$ Key Laboratory of Gastroenterology of Zhejiang Province, \\ Zhejiang Provincial People's Hospital, Hangzhou, Zhejiang 310014, P.R. China
}

Received December 21, 2013; Accepted March 12, 2014

DOI: $10.3892 / \mathrm{ijmm} .2014 .1705$

\begin{abstract}
Type II alveolar epithelial cells (AECII) play a key role in maintaining normal alveolar homeostasis and repair. AECII derived from exogenous stem cells may provide novel treatment options for distal lung diseases. In this study, to explore whether amniotic fluid-derived mesenchymal stem cells (AFMSCs) may be induced to differentiate into AECII in vitro, AFMSCs were isolated from 15 independent samples of amniotic fluid, in which CD29, CD44, CD73, CD90, CD105 and CD166 were significantly expressed, but the expression of CD14, CD19, CD34 and CD45 was negative. Octamer-binding transcription factor 4 (OCT4) at both the mRNA and protein level was also significantly expressed in the AFMSCs. We demonstrate that AFMSCs cannot be induced to differentiate into AECII using KnockOut ${ }^{\mathrm{TM}}$ serum replacement (KOSR) only. Surfactant protein (SP)A and SPC mRNA expression in the differentiated AFMSCs was significantly induced by the appropriate combination of KOSR, activin A and small airway basal medium (SABM). However, SPA and SPC expression was negative with an inappropriate induction. Lamellar bodies were observed only in the cells which were appropriately induced by KOSR, activin A and SABM. Thus, these results indicate that AFMSCs may be induced to differentiate into AECII-like cells in vitro with the use of the appropriate induction medium, including KOSR, activin A and SABM, suggesting that that AFMSCs have the potential for use in lung regenerative therapy.
\end{abstract}

\section{Introduction}

The mammalian alveolar epithelium is composed of type I alveolar epithelial cells (AECI) and type II alveolar epithelial cells (AECII). AECI are broad and flat cells that cover $95 \%$ of the alveolar surface and comprise $40 \%$ of the alveolar

Correspondence to: Dr Yaqing Li, Department of Respiratory Medicine, Zhejiang Provincial People's Hospital, 158 Shangtang Road, Hangzhou, Zhejiang 310014, P.R. China

E-mail: lidoctor03@126.com

Key words: mesenchymal stem cells, amniotic fluid, alveolar epithelial cells, chronic obstructive pulmonary disease epithelium, which is important for gas exchange. AECII are cuboidal cells characterized by the distinct morphological appearance of lamellar bodies, which occupy only $5 \%$ of the alveolar surface and comprise $60 \%$ of the alveolar epithelium. AECII synthesize, secrete and recycle lung surfactant, which includes surfactant protein (SP)A, SPB, SPC and SPD, reducing surface tension and preventing the collapse of the alveolus (1). AECII also provide pulmonary host defense by synthesizing and secreting numerous cytokines and interleukins that affect lymphocyte, macrophage, and neutrophil functions. Moreover, AECII act as progenitor cells for AECI in the alveoli, and play a key role in maintaining normal alveolar homeostasis and lung repair (2). The inadequate, delayed, or impaired re-epithelialization of the injured alveolus is known as a key factor in the pathogenesis of several life-threatening pulmonary diseases, such as chronic obstructive pulmonary disease (COPD) (3) and acute respiratory distress syndrome (ARDS) (4). A variety of methods for isolating human AECII have been published and some of their properties have been described (5-7); however, it still remains unclear as to how to maintain the proliferation and phenotype of the AECII. A number of difficulties still exist in obtaining an adequate tissue supply to isolate human AECII. Human embryonic stem cells (ESCs) are pluripotent stem cells derived from embryos at the blastocyst stage. ESCs can proliferate indefinitely while maintaining their capacity to differentiate into a great variety of specialized cell types $(8,9)$. ESCs have been induced to differentiate into AECII in vitro and in vivo $(10,11)$. Furthermore, the transplantation of human ESC-derived AECII has been shown to abrogate acute lung injury in mice (12). Thus, AECII derived from exogenous stem cells may provide novel treatment options for COPD.

However, the acquirement of human ESCs implies the destruction of human embryo, which results in obvious ethical debates (13). Human amniotic fluid stem cells (AFSCs) can be isolated from discarded amniocentesis specimens or postpartum amniotic fluid, which is not subject to teratocarcinoma formation and serious ethical issues $(14,15)$. AFSCs possess not only high proliferation potential in vitro, but also a wide range of differentiation potential into cell types from all 3 embryonic germ layers, such as adipocytes, chondrocytes, osteocytes, hepatocytes, neural cells and cardiomyocytes (16-18). Hence, AFSCs are considered new pluripotent stem cells between ESCs and adult stem cells. Their characteristics, including pluripotency, high proliferation rates, multi-differentiation capabilities and 
lack of ethical issues associated with the retrieval of these cells render them an attractive source for cell therapy, drug discovery and toxicology screening (14-18). Certain studies have demonstrated that human AFSCs can integrate into the murine lung and differentiate into pulmonary lineages following lung injury in vivo (19). However, it remains unknown as to whether amniotic fluid-derived mesenchymal stem cells (AFMSCs) can be induced to differentiate into AECII in vitro. Thus, in the present study, we investigated the potential of AFMSC differentiation into AECII in vitro using KnockOut ${ }^{\mathrm{TM}}$ serum replacement (KOSR), activin A and small airway basal medium (SABM).

\section{Materials and methods}

Isolation and culture of AFMSCs. Fifteen independent samples of amniotic fluid ( $20 \mathrm{ml}$ each) were obtained from pregnant women at 16-20 weeks of pregnancy who underwent amniocentesis for fetal karyotyping. The samples were filtered through a 200 mesh filter and centrifuged for $5 \mathrm{~min}$ at $1,200 \mathrm{rpm}$. The cells derived from the amniotic fluid were resuspended in low glucose Dulbecco's modified Eagle's medium (DMEM; Gibco, Carlsbad, CA, USA) containing $5 \mathrm{mg} / \mathrm{l}$ basic fibroblast growth factor (bFGF; Sigma, St. Louis, MO, USA) and 10\% fetal bovine serum (FBS; HyClone, Logan, UT, USA), and maintained in 6-well plates. When the cells were incubated for 3 days at $37^{\circ} \mathrm{C}$ in a humidified atmosphere of 5\% carbon dioxide and $95 \%$ air, the supernatants were removed and the cell clones in spindle-shaped growth were selected to other wells by cell scrapers. When the cells reached $80-90 \%$ confluence, they were digested using $0.25 \%$ trypsin (Sigma) and incubated in a $25 \mathrm{~cm}^{2}$ cell culture flask (Corning Inc.-Life Sciences, Oneonta, NY, USA), labeled as P1 (the first generation). The surface antigens of the AFMSCs (P4) were detected by flow cytometry with monoclonal antibodies as follows: phycoerythrin (PE)-conjugated antibodies against CD29,CD73, CD105 and CD166, and fluorescein isothiocyanate (FITC)-conjugated antibodies against CD14, CD19, CD34, CD44, CD45 and CD90 (BD Biosciences, San Diego, CA, USA). The related isotype control was used as a negative control. The results were analyzed using a flow cytometer (Guava EasyCyte; Millipore, Billerica, MA, USA). Octamer-binding transcription factor 4 (OCT4) mRNA expression in the AFMSCs was detected by reverse transcription-polymerase chain reaction (RT-PCR). The primers were designed as follows (Invitrogen, Shanghai, China): OCT4 (GenBank: DQ486513.1), 5'-CGTG AAGCTGGAGAAGGAGAAGCTG-3' (sense) and 5'-CAAGGG CCGCAGCTTACACATGTTC-3' (antisense); and beta-actin $(A C T B), 5^{\prime}$-GGCACCACACCTTCTACAATGA-3' (sense) and 5'-TCAGGAGGAGCAGCAATGATCTTG-3' (antisense). The protein expression of OCT4 was detected by western blot analysis. Human embryonic stem cells (hES3; ES Cell International, Melbourne, Australia) were used as positive controls and human lung fibroblasts (HFL1; ATCC, Manassas, VA, USA) were used as negative controls. The protocol was approved by the Ethics Committee of Zhejiang Provincial People's Hospital and each patient signed written informed consent.

Differentiation of AFMSCs in vitro. The AFMSCs (P4) were detached and dissociated into single cells with a mixture of $0.25 \%$ trypsin (Sigma) $/ 0.02 \%$ EDTA- $\mathrm{Na}_{2}$ in phosphate-buffered saline (PBS). The AFMSCs were seeded into 24-well lowadherence tissue culture plates (Corning Inc.-Life Sciences) at the concentration of $2 \times 10^{7} / 1 /$ well. They were then divided into 4 groups, named groups I-IV. The AFMSCs were induced to differentiate in vitro using KOSR (Invitrogen, Carlsbad, CA, USA), activin A (PeproTech, Rocky Hill, NJ, USA) and SABM (Lonza, Basel, Switzerland) by different methods. In group I, the AFMSCs were cultured for 3 days suspended in DMEM (Gibco) containing 10\% KOSR firstly, and then transferred onto adherent agarose-coated plates. The adherent cells were cultured for 4 days in DMEM containing 10\% KOSR with $100 \mathrm{mg} / \mathrm{l}$ activin A in a humidified atmosphere of 5\% carbon dioxide and $95 \%$ air at $37^{\circ} \mathrm{C}$. The cells were then cultured for 10 days in DMEM containing 15\% KOSR. In group II, the AFMSCs were cultured for 3 days suspended in DMEM containing 10\% KOSR firstly, and then adherently cultured for 4 days in DMEM containing 10\% KOSR. The cells were then cultured for 10 days in DMEM containing 15\% KOSR. In group III, the AFMSCs were cultured for 3 days suspended in DMEM containing $10 \%$ KOSR with $100 \mathrm{mg} / \mathrm{l}$ activin A firstly, and then cultured for 4 days adherently in DMEM containing $10 \%$ KOSR. The cells were then cultured for 10 days in DMEM containing 15\% KOSR. In group IV, the AFMSCs were cultured for 3 days suspended in DMEM containing $10 \%$ KOSR with $100 \mathrm{mg} / \mathrm{l}$ activin A firstly, and then adherently cultured for 4 days in DMEM containing 10\% KOSR with $100 \mathrm{mg} / \mathrm{l}$ activin A. The cells were then cultured for 10 days in DMEM containing 15\% KOSR. Finally, all the cells were cultured for 14 days in SABM.

Quantitative reverse transcription PCR. Total RNA was extracted from the cells using TRIzol reagent (Invitrogen, Carlsbad, CA, USA) according to the instructions of the manufacturer and dissolved in nuclease-free water. The final RNA concentrations were determined using a spectrophotometer. Total RNA was reverse transcribed using MMLV reverse transcriptase (Promega, Madison, WI, USA) at $42^{\circ} \mathrm{C}$ for $60 \mathrm{~min}$ and $70^{\circ} \mathrm{C}$ for $10 \mathrm{~min}$. Real-time PCR analysis was carried out using SYBR-Green Real-Time PCR Master Mix (Toyobo, Osaka, Japan) and real-time PCR amplification equipment. The thermal profile consisted of one cycle at $95^{\circ} \mathrm{C}$ for $15 \mathrm{~min}$, followed by 45 cycles at $95^{\circ} \mathrm{C}$ for $15 \mathrm{sec}$ and $60^{\circ} \mathrm{C}$ for $30 \mathrm{sec}$. The primers used were as follows (Invitrogen, Shanghai, China): SPA forward, 5'-CCTATCATTTGCCAA GAACC-3' and reverse, 5'-CAGGAAGATGGGTTTGGAT-3'; $S P C$ forward, 5'-CAACGGGAAAGGAAACGC-3' and reverse, 5'-GCTGCTTTATTCTGTTGTGGC-3'; and ACTB forward, 5'-CTACAATGAGCTGCGTGTGGC-3' and reverse, 5'-CAGG TCCAGACGCAGGATGGC-3'. The mRNA expression of $S P A$ and $S P C$ was determined by normalization of the threshold (Ct) cycle of the target genes to that of the mRNA expression of $A C T B$ for each sample. The $\triangle \mathrm{Ct}$ value was determined using the following equation: $(\Delta \mathrm{Ct})=(\mathrm{Ct}$ of the target genes) $-(\mathrm{Ct}$ of $A C T B)$. The $\triangle \Delta C$ t value was used to find the relative expression of the target genes according to the following formula: relative expression $0=2^{-\Delta \Delta \mathrm{Ct}}$. Data were analyzed using the $2^{-\Delta \Delta \mathrm{Ct}}$ method. A549 lung epithelial cells (Shanghai Institute of Biology, Chinese Academy of Sciences, Shanghai, China) were used as the positive controls. Undifferentiated AFMSCs were used as the negative controls. 

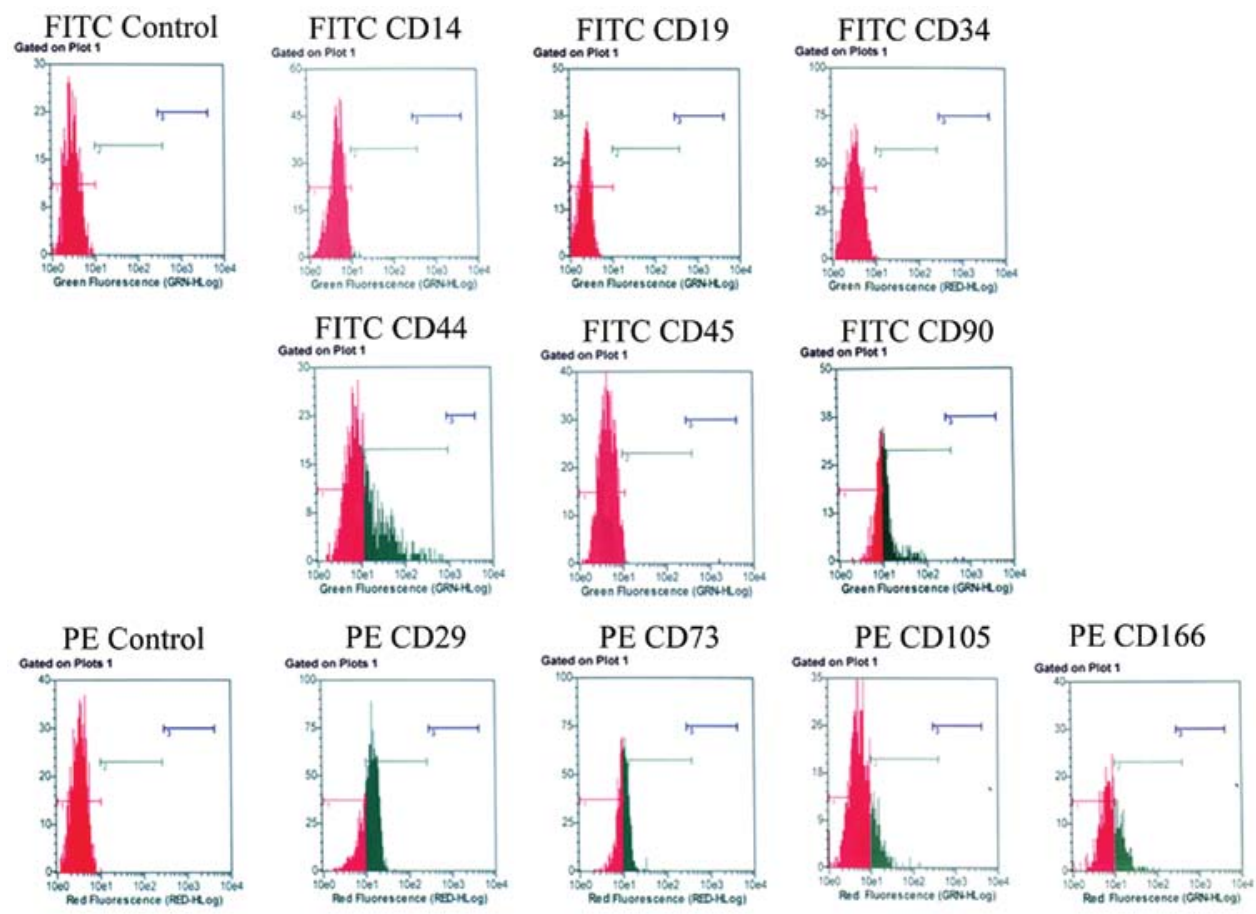

Figure 1. Phenotypes of amniotic fluid-derived mesenchymal stem cells (AFMSCs). The phenotypes of AFMSCs were detected by flow cytometric analyses The related isotype control was used as a negative control. CD29, CD44, CD73, CD90, CD105 and CD166 were significantly expressed in the AFMSCs (P4), but the expression of CD14, CD19, CD34 and CD45 was negative in the AFMSCs.

Western blot analysis. The cells were lysed in ice-cold lysis buffer [50 mmol/1 Tris (pH 7.5), $150 \mathrm{mmol} / \mathrm{l} \mathrm{NaCl}, 1 \mathrm{mmol} / 1$ EDTA, $10 \mathrm{mmol} / 1 \mathrm{Na}_{2} \mathrm{P}_{2} \mathrm{O}_{7}, 100 \mathrm{mmol} / \mathrm{l} \mathrm{NaF}$ and $1 \%$ Triton X-100] and protease inhibitor cocktail ( $1 \mathrm{mmol} / \mathrm{l}$ phenylmethylsulfonyl fluoride, $1 \mathrm{mg} / \mathrm{l}$ aprotinin and $1 \mathrm{mg} / \mathrm{l}$ leupeptin). The protein concentration was measured using a protein assay kit (Micro BCA; Pierce, Rockford, IL, USA). Equal amounts of cell lysates containing $10 \mu \mathrm{g}$ protein were incubated for $5 \mathrm{~min}$ in boiling water. Proteins were separated by $8 \%$ sodium dodecyl sulfate polyacrylamide gel electrophoresis and then electroblotted onto Hybond-ECL nitrocellulose membranes. The membranes were blocked with TBS solution containing $5 \%$ skim milk at room temperature for $1 \mathrm{~h}$. The membranes were then incubated with primary antibodies for $1 \mathrm{~h}$ at room temperature on an orbital shaker. The membranes were then washed 5 times, for $5 \mathrm{~min}$ each, in Tris-buffered saline with Tween-20 (TBST) and incubated in diluted peroxidase-conjugated immunopure goat anti-rabbit IgG $(\mathrm{H}+\mathrm{L})$ (Pierce) for $1 \mathrm{~h}$ at room temperature on an orbital shaker. After the membranes were again washed 5 times for 5 min each in TBST, the proteins were visualized with an enhanced chemiluminescence solution (Amersham Pharmacia Biotech, Buckinghamshire, UK). The images were developed on X-ray film and the band densities were analyzed with a UVP-GDS8000 gel analysis system. The same membranes were stripped and blotted with anti- $\beta$-actin antibodies, which provided a loading control. The primary antibodies were obtained from the following sources: OCT4 antibody, SPA antibody and SPC antibody from Abcam (Cambridge, MA, USA), and $\beta$-actin (N-21) antibody from Santa Cruz Biotechnology, Inc. (Santa Cruz, CA, USA). Protein markers were obtained from Cell Signaling Technology (Danvers, MA, USA).
Immunofluorescence. The differentiated AFMSCs were fixed for $20 \mathrm{~min}$ at $4^{\circ} \mathrm{C}$ with $4 \%$ paraformaldehyde (Merck, Darmstadt, Germany). The cells were blocked for $20 \mathrm{~min}$ at $37^{\circ} \mathrm{C}$ with $5 \%$ goat serum (diluted in TBS and 0.25\% Triton X-100; Sigma), followed by incubation for $2 \mathrm{~h}$ at $37^{\circ} \mathrm{C}$ with a polyclonal rabbit anti-human SPA and SPC antibody (1:500; BD Biosciences). The cells were then rinsed twice for $5 \mathrm{~min}$ with PBS, and further incubated for $1 \mathrm{~h}$ at $37^{\circ} \mathrm{C}$ with the secondary antibody FITCconjugated goat anti rabbit IgG (1:250; BD Biosciences). Each antibody was diluted in PBS with $0.25 \%$ Triton X-100. The cell nuclei were then counterstained with $1 \mathrm{~g} / 1$ 4',6-diamino-2-phenylindole (DAPI; Sigma) in PBS for 5 min and covered with Immu-Mount (Thermo Electron Corp., Waltham, MA, USA). Finally, all the slides were analyzed using an IX71 fluorescence microscope (Olympus, Tokyo, Japan).

Electron microscopy. The cells were fixed for $1 \mathrm{~h}$ in $2.5 \%$ glutaraldehyde and $0.1 \mathrm{mmol} / 1$ cacodylate buffer, $\mathrm{pH}$ 7.4. After a brief rinse in $0.2 \mathrm{mmol} / \mathrm{l}$ buffer, the cells were postfixed for $2 \mathrm{~h}$ in $1 \%$ osmium tetroxide in $0.1 \mathrm{mmol} / 1$ cacodylate buffer, $\mathrm{pH}$ 7.4. The cells were dehydrated in 90,95 and $100 \%$ hydroxypropyl methacrylate and embedded in Epon-Araldite. Ultrathin serial sections $(100 \mathrm{~nm})$ were obtained by an ultramicrotome (RMC, Tucson, AZ, USA) equipped with a diamond knife. The sections were stained with uranyl acetate and lead citrate, and then observed under a transmission electron microscope (Hitachi, Tokyo, Japan).

Statistical analysis. Data were analyzed for statistical significance by one-way analysis of variance (ANOVA), paired-sample t-tests, and multiple comparisons in ANOVA were analyzed using the Student-Newman-Keuls test. Data are expressed as 

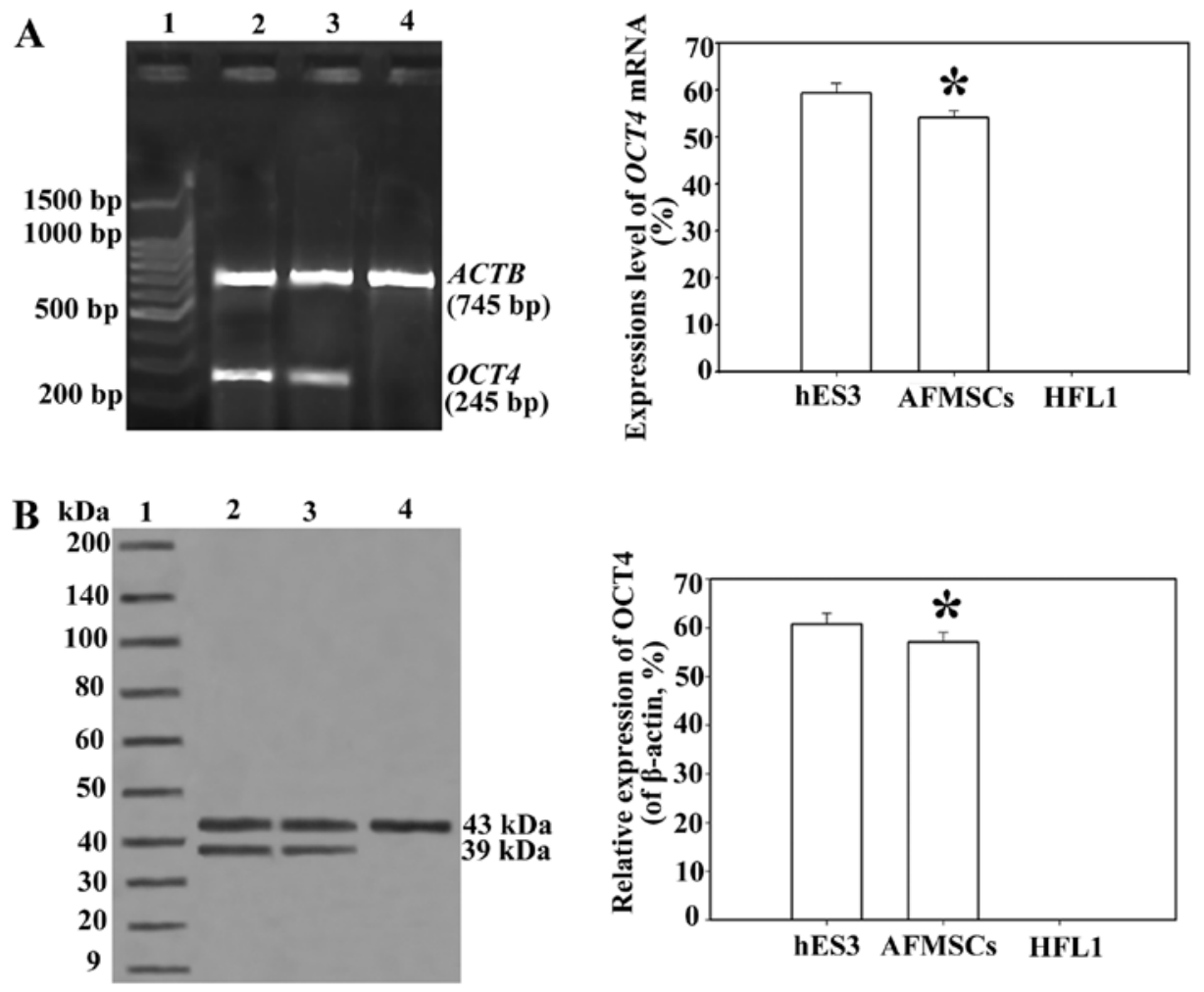

Figure 2. Octamer-binding transcription factor 4 (OCT4) expression in amniotic fluid-derived mesenchymal stem cells (AFMSCs). (A) OCT4 mRNA expression in the AFMSCs was detected by RT-PCR. The mRNA expression of OCT4 was evaluated according to the OCT4 mRNA/ACTB mRNA ratio. (B) The protein expression of OCT4 in the AFMSCs was detected by western blot analysis. The protein expression of OCT4 was evaluated according to the ratio of OCT4/ $\beta$ actin. (A and B) Lane 1 respresents the DNA ladder marker or protein marker, respectively. Lane 2, positive control with hES3 cells. "P<0.05 vs. the expression of OCT4 in hES3 cells; data are the means \pm standard deviation (SD) of 8 separate experiments.

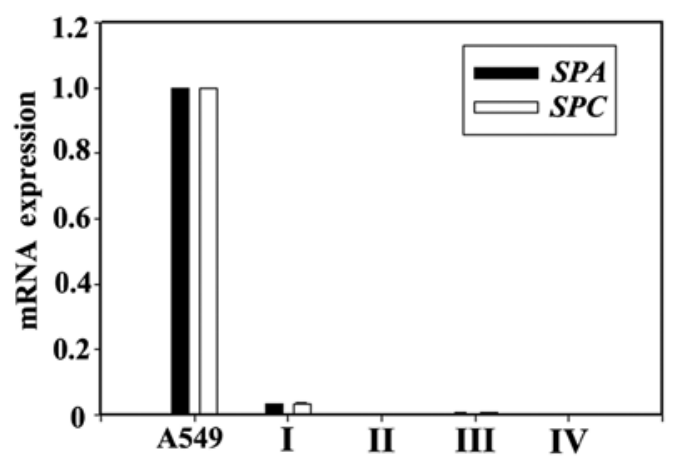

Figure 3. Surfactant protein $(S P) A$ and $S P C$ mRNA expression in differentiated amniotic fluid-derived mesenchymal stem cells (AFMSCs). $S P A$ and $S P C$ mRNA expression in differentiated AFMSCs was detected by quantitative reverse transcription polymerase chain reaction (PCR). The relative expression of $S P A$ and $S P C$ mRNA was analyzed using the values of $2^{-\Delta \Delta \mathrm{Ct}}$. The mRNA expression of $S P A$ and $S P C$ in A549 lung epithelial cells was used as a positive control. $S P A$ and $S P C$ mRNA expression was not detected in the undifferentiated AFMSCs which were used as negative controls. Data are the means \pm standard deviation (SD) of 3 separate experiments.

the means \pm standard deviation (SD). Values of $\mathrm{P}<0.05$ were considered to indicate statistically significant differences.

\section{Results}

Phenotypic characterization of AFMSCs. The surface antigenic characteristics of the AFMSCs were analyzed using flow cytometry. The results revealed that the expression of surface

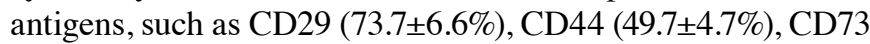
$(53.0 \pm 3.6 \%), \mathrm{CD} 90(54.4 \pm 4.35 \%), \mathrm{CD} 105(22.5 \pm 2.7 \%)$ and CD166 (22.7 $\pm 3.6 \%)$ was positive; however, the expression of CD14 (0.25 $\pm 0.02 \%)$, CD19 (0\%), CD34 (0\%) and CD45 (0\%) was negative (Fig. 1). OCT4, at both the mRNA and protein level was significantly expressed in the AFMSCs $(\mathrm{P}<0.05)$ (Fig. 2).

mRNA expression of SPA and SPC in differentiated AFMSCs. The mRNA expression of SPA and $S P C$ in the A549 lung epithelial cells (positive controls) was strongly positive. The mRNA expression of SPA and SPC was not detected in the undifferentiated AFMSCs. When the AFMSCs were induced to differentiate using KOSR only, the mRNA expression of $S P A$ and SPC was negative (group II). When the AFMSCs were induced to differentiate using a combination of KOSR, activin A and SABM, the mRNA expression of SPA and $S P C$ (group III and IV) was still almost undetectable. The mRNA expression of SPA and SPC in the cells in group I was significantly induced, and was the highest amongst all the groups $(\mathrm{P}<0.05)$ (Fig. 3).

Protein expression of SPA and SPC in differentiated AFMSCs. When the AFMSCs were induced to differentiate for 31 days, the protein expression of SPA and SPC was detected by western blot analysis and immunofluorescence. The results 

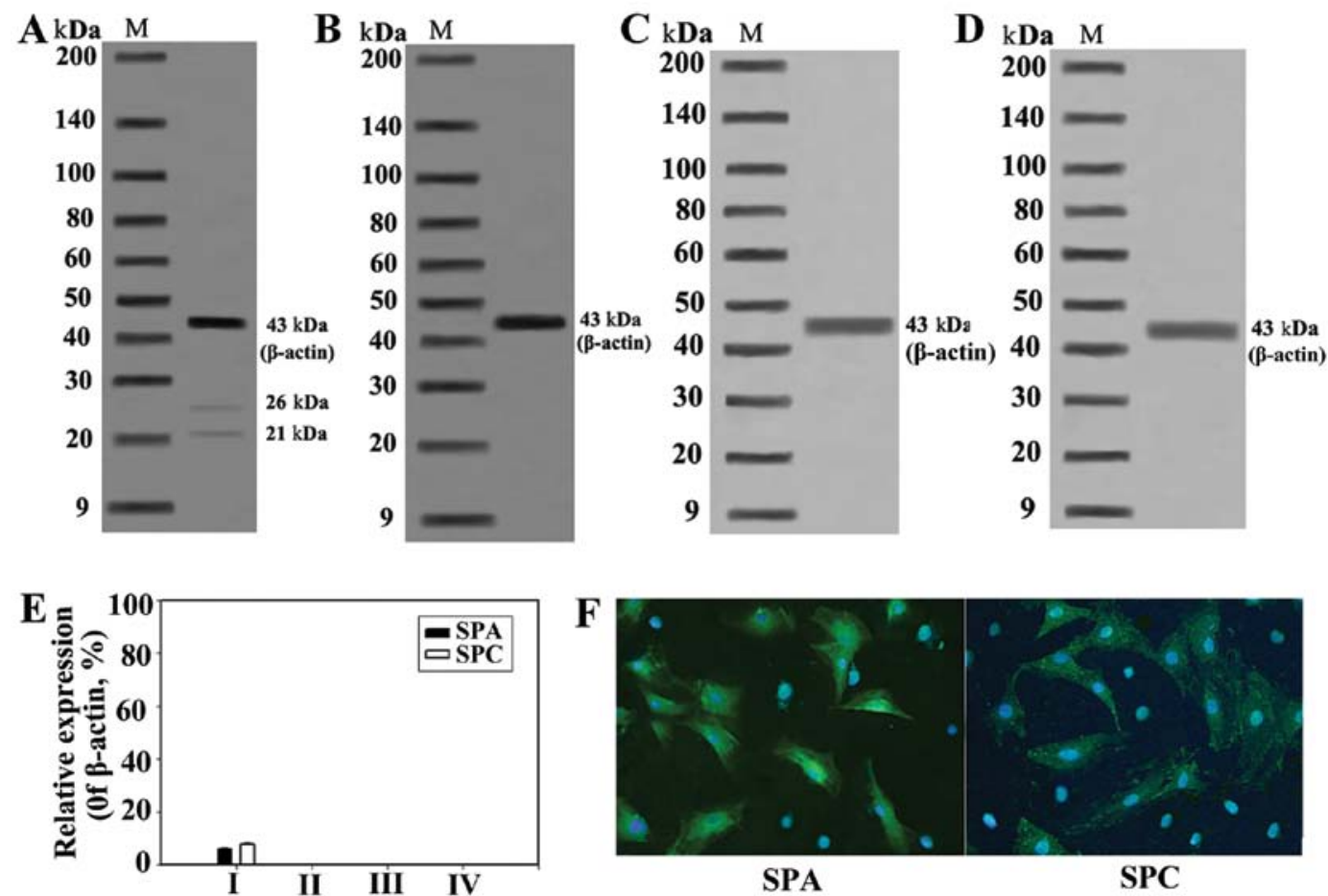

Figure 4. The protein expression of surfactant protein (SP)A and SPC in differentiated amniotic fluid-derived mesenchymal stem cells (AFMSCs). The AFMSCs of all groups were respectively induced to differentiate in vitro as described in Materials and methods. SPA and SPC expression in differentiated AFMSCs was respectively detected by western blot analysis and immunofluorescence. (A) Group I; (B) group II; (C) group III ; (D) group IV; M, protein marker. (E) SPA and SPC expression in group I was significantly induced, but negative in the other groups. (F) SPA and SPC expression in differentiated AFMSCs of group I was detected by immunofluorescence (magnification, x200). SPA and SPC expression in group I was positive, but the protein expression of SPA and SPC could not be detected in groups II-IV.
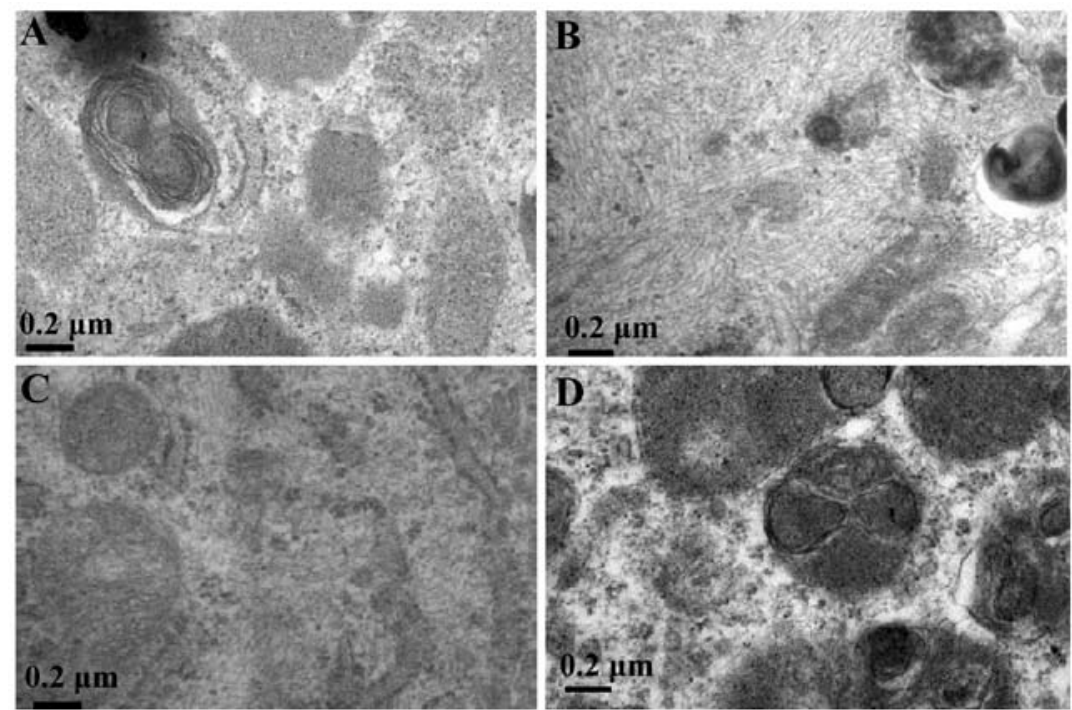

Figure 5. Ultrastructures of differentiated amniotic fluid-derived mesenchymal stem cells (AFMSCs). (A) Group I; (B) group II; (C) group III; (D) group IV. Lamellar bodies were observed in the differentiated AFMSCs in group I. Lamellar bodies could not be observed in groups II-IV.

revealed that the expression of SPA and SPC in group I was significantly induced $(\mathrm{P}<0.05)$, but was negative in the cells in the other groups (Fig. 4), which indicates that the AFMSCs can be induced to differentiate into AECII-like cells under specific conditions.

Ultrastructural evidence of AFMSCs differentiating into AECII-like cells. When the AFMSCs were induced to differ- entiate for 31 days using KOSR, activin A and SABM, lamellar bodies that contained pulmonary surfactant proteins and lipids were observed in the differentiated AFMSCs in group I under a transmission electron microscope (Fig. 5). However, lamellar bodies were not observed in the cells in groups II-IV. This indicates that AFMSCs can differentiate into AECII-like cells by using the appropriate induction medium with KOSR, activin A and SABM. 


\section{Discussion}

AFMSCs are pluripotent stem cells, which can be isolated from discarded amniocentesis specimens or post-partum amniotic fluid $(14,20)$. Previous studies have demonstrated that AFMSCs express mesenchymal stem cell markers, such as CD90, CD105, CD73 and CD166, but not do not express the hematopoietic markers, CD45, CD34 and CD14 (20-22). In the present study, AFMSCs significantly expressed surface antigens, such as CD29, CD44, CD73 and CD90, but did not express CD14, CD19, CD34 and CD45, which was consistent with the study of Tsai et al (14). The potency of amniotic fluidderived stem cells in differentiating into cell types from all 3 embryonic germ layers, including endothelial cells, chondrocytes, adipocytes, hepatocytes, neuronal cells and myocytes seems to be between ESCs and adult stem cells (16-18,23). The POU transcription factor, OCT4, is a specific marker for embryonal carcinoma cells, embryonic germ cells and embryonic stem cells (24). In the present study, OCT4 was significantly expressed in the AFMSCs, which indicated that the AFMSCs have pluripotent characteristics. Thus, their pluripotency, lack of ethical issues associated with their acquirement and the lack of teratoma formation when injected in vivo render them attractive candidates for stem cell sources in the field of lung regeneration (14-18).

As progenitors of AECI, AECII characterized by the unique ability to synthesize and secrete SPC and the distinct morphological appearance of lamellar bodies play important roles in lung tissue repair (1). Severe damage to or the loss of AECII may result in a considerable vulnerability of the alveolus and the impairment of alveolar repair (25-27). AECII derived from exogenous stem cells are a promising source of cells which may be used therapeutically to treat distal lung diseases, including COPD. However, to date, there are still no reports on the differentiation of AFMSCs into AECII in vitro $(16,28)$. Small airway growth medium (SAGM) composed of SABM and a commercial cocktail of growth factors is a medium designed for the maintenance and growth of mature distal airway epithelial cells. However, certain studies have shown that SABM significantly improves distal lung epithelial differentiation from mouse ESCs compared with SAGM (29). Thus, in the present study, SABM was used to induce AFMSC differentiation in vitro in place of SAGM. KOSR is a defined medium supplement designed to directly replace FBS in ESC culture medium. Certain studies have shown that definitive endoderm differentiation is improved (30) and human ESCs are induced to differentiate into AECII by the replacement of FBS with KOSR (11). In the present study, AFMSCs failed to differentiate into AECII when induced with only KOSR. Activin A belongs to the transforming growth factor- $\beta$ (TGF- $\beta$ ) family of growth and differentiation factors (31). Studies on amphibians and have mice shown that high activity of activin signaling is necessary to specify the endoderm germ layer $(32,33)$. It has also been demonstrated that activin A can increase the expression of early distal lung epithelial markers, enhance endoderm formation and accelerate early lung epithelial differentiation (29). In the present study, when the AFMSCs were induced to differentiate for 31 days by KOSR, activin A and SABM, the mRNA and protein expression of SPA and SPC was strongly positive in the differentiated AFMSCs in group I, and lamellar bodies were also observed in the differentiated AFMSCs. However, negative results were observed for the other cell groups, which suggests that the differentiation of AFMSCs is precisely controlled by both time and space. In conclusion, AFMSCs can be induced to differentiate into AECII-like cells in vitro using a combination of KOSR, activin A and SABM under the appropriate conditions, which indicates that AFMSCs have the potential for use in lung regenerative therapy. However, the exact mechanisms of AFMSC differentiation into AECII reamin unknown, and thus, further studies are required in the near future.

\section{Acknowledgements}

This study was supported by a grant from the National Natural Science Foundation of China (no. 81000016).

\section{References}

1. Whitsett JA, Wert SE and Weaver TE: Alveolar surfactant homeostasis and the pathogenesis of pulmonary disease. Annu Rev Med 61: 105-119, 2010

2. Hoffman AM and Ingenito EP: Alveolar epithelial stem and progenitor cells: emerging evidence for their role in lung regeneration. Curr Med Chem 19: 6003-6008, 2012.

3. Tsuji T, Aoshiba K and Nagai A: Alveolar cell senescence exacerbates pulmonary inflammation in patients with chronic obstructive pulmonary disease. Respiration 80: 59-70, 2010.

4. Miyake Y, Kaise H, Isono K, Koseki H, Kohno K and Tanaka M: Protective role of macrophages in noninflammatory lung injury caused by selective ablation of alveolar epithelial type II Cells. J Immunol 178: 5001-5009, 2007.

5. Corti M, Brody AR and Harrison JH: Isolation and primary culture of murine alveolar type II cells. Am J Respir Cell Mol Biol 14: 309-315, 1996.

6. Ehrhardt C, Kim KJ and Lehr CM: Isolation and culture of human alveolar epithelial cells. Methods Mol Med 107: 207-216, 2005.

7. Fujino N, Kubo H, Suzuki T, et al: Isolation of alveolar epithelial type II progenitor cells from adult human lungs. Lab Invest 91: 363-378, 2011.

8. Gaur M, Ritner C, Sievers R, Pedersen A, Prasad M, Bernstein HS and Yeqhiazarians Y: Timed inhibition of p38MAPK directs accelerated differentiation of human embryonic stem cells into cardiomyocytes. Cytotherapy 12: 807-817, 2010.

9. Muguruma K and Sasai Y: In vitro recapitulation of neural development using embryonic stem cells: from neurogenesis to histogenesis. Dev Growth Differ 54: 349-357, 2012.

10. Samadikuchaksaraei A and Bishop AE: Derivation and characterization of alveolar epithelial cells from murine embryonic stem cells in vitro. Methods Mol Biol 330: 233-248, 2006.

11. Wang D, Haviland DL, Burns AR, Zsiqmond E and Wetsel RA: A pure population of lung alveolar epithelial type II cells derived from human embryonic stem cells. Proc Natl Acad Sci USA 104: 4449-4454, 2007.

12. Wang D, Morales JE, Calame DG, Alcorn JL and Wetsel RA: Transplantation of human embryonic stem cell-derived alveolar epithelial type II cells abrogates acute lung injury in mice. Mol Ther 18: 625-634, 2010.

13. Sugarman J: Human stem cell ethics: beyond the embryo. Cell Stem Cell 2: 529-533, 2008.

14. Tsai MS, Lee JL, Chang YJ and Hwang SM: Isolation of human multipotent mesenchymal stem cells from second-trimester amniotic fluid using a novel two-stage culture protocol. Hum Reprod 19: 1450-1456, 2004.

15. Sessarego N, Parodi A, Podestà M, et al: Multipotent mesenchymal stromal cells from amniotic fluid: solid perspectives for clinical application. Haematologica 93: 339-346, 2008.

16. Joo S, Ko IK, Atala A, Yoo JJ and Lee SJ: Amniotic fluid-derived stem cells in regenerative medicine research. Arch Pharm Res 35: 271-280, 2012.

17. Hartmann K, Raabe O, Wenisch S and Arnhold S: Amniotic fluid derived stem cells give rise to neuron-like cells without a further differentiation potential into retina-like cells. Am J Stem Cells 2: 108-118, 2013. 
18. Sun H, Feng K, Hu J, Soker S, Atala A and Ma PX: Osteogenic differentiation of human amniotic fluid-derived stem cells induced by bone morphogenetic protein-7 and enhanced by nanofibrous scaffolds. Biomaterials 31: 1133-1139, 2010.

19. Carraro G, Perin L, Sedrakyan S, et al: Human amniotic fluid stem cells can integrate and differentiate into epithelial lung lineages. Stem Cells 26: 2902-2911, 2008.

20. Perin L, Sedrakyan S, Da Sacco S and De Filippo R: Characterization of human amniotic fluid stem cells and their pluripotential capability. Methods Cell Biol 86: 85-99, 2008.

21. De Coppi P, Bartsch G Jr, Siddiqui MM, et al: Isolation of amniotic stem cell lines with potential for therapy. Nat Biotechnol 25 100-106, 2007.

22. Roubelakis MG, Trohatou $\mathrm{O}$ and Anagnou NP: Amniotic fluid and amniotic membrane stem cells: marker discovery. Stem Cells Int 2012: 107836, 2012.

23. Benavides OM, Petsche JJ, Moise KJ Jr, Johnson A and Jacot JG: Evaluation of endothelial cells differentiated from amniotic fluid-derived stem cells. Tissue Eng Part A 18: 1123-1131, 2012.

24. Tondeur S, Assou S, Nadal L, Hamamah S and De Vos J: Biology and potential of human embryonic stem cells. Ann Biol Clin (Paris) 66: 241-247, 2008 (In French).

25. Sisson TH, Mendez M, Choi K, et al: Targeted injury of type II alveolar epithelial cells induces pulmonary fibrosis. Am J Respir Crit Care Med 181: 254-263, 2010.
26. Kuwano K: Epithelial cell apoptosis and lung remodeling. Cell Mol Immunol 4: 419-429, 2007.

27. Zhao CZ, Fang XC, Wang D, Tang FD and Wang XD: Involvement of type II pneumocytes in the pathogenesis of chronic obstructive pulmonary disease. Respir Med 104: 1391-1395, 2010.

28. Antonucci I, Pantalone A, Tete S, Salini V, Borlongan CV, Hess D and Stuppia L: Amniotic fluid stem cells: a promising therapeutic resource for cell-based regenerative therapy. Curr Pharm Des 18: 1846-1863, 2012.

29. Rippon HJ, Polak JM, Qin M and Bishop AE: Derivation of distal lung epithelial progenitors from murine embryonic stem cells using a novel three-step differentiation protocol. Stem Cells 24: 1389-1398, 2006

30. Kubo A, Shinozaki K, Shannon JM, et al: Development of definitive endoderm from embryonic stem cells in culture. Development 131: 1651-1662, 2004.

31. Massagué J and Gomis RR: The logic of TGFbeta signaling. FEBS Lett 580: 2811-2820, 2006.

32. Cao Y, Siegel D and Knöchel W: Xenopus POU factors of subclass V inhibit activin/nodal signaling during gastrulation. Mech Dev 123: 614-625, 2006.

33. Hansson M, Olesen DR, Peterslund JM, et al: A late requirement for Wnt and FGF signaling during activin-induced formation of foregut endoderm from mouse embryonic stem cells. Dev Biol 330: 286-304, 2009. 